\title{
Self - Organizing and Coverage Recovery Techniques for Wireless Sensor Networks
}

\author{
M. Pravin \\ Asst Prof (Sr. Gr), Department of ECE, \\ Sri Ramakrishna Institute of Technology, \\ Coimbatore, India.
}

\author{
T. V. P. Sundararajan, PhD \\ Professor, Department of ECE, \\ Bannari Amman Institute of Technology, \\ Sathyamangalam, India.
}

\begin{abstract}
The major issue of a Wireless Sensor Network (WSN), is to provide sufficient and uninterrupted coverage, to provide this heterogeneous sensors are used. Sensors have wide range of sensing capability is called heterogeneous sensors. In order to enable Sensor-Self Deployment these sensor nodes were made movable. The protocol used to increase the efficiency of the system and to reduce the power consumption is coverage aware sensor automation (CASA).

The CASA protocol comprises of two algorithms. First one is sensor self-organizing algorithm (SSOA) and the second algorithm is enhanced virtual forces algorithm with boundary forces (EVFA-B). The EVFA-B states that each sensor node will have attractive and repulsive forces on every other sensor based of distance thresholding. The resulting force is used to place the sensor node to proper location in order to achieve the energy efficiency. The SSOA is used when there is a sudden or unexpected failure of sensor node. It performs local repair by repositioning the sensor. The performance of CASA is evaluated in terms of moving energy consumption and network self-healing capacity.
\end{abstract}

\section{Keywords}

ISM, WSN, EVFA- B, SSOA and CASA

\section{INTRODUCTION}

The sensor networks were self-organized to monitor physical or environmental conditions and pass their data through the network to a server [3].

The development of this new technology was motivated by numerous applications with high potential such as military, medical, environmental etc.

\subsection{Power Management}

A good power management technique can increase the lifetime of a sensor node. Many researchers doing their research to increase the lifetime of a sensor node because all the system needs longer lifetime to meet the functional requirements.

The simulation level results minimizes the communications by providing sleep/wakeup schedules because transmission and listening for messages leads to more energy consumption.

\subsection{Energy Consumption}

The energy consumption should be minimized with the goal of increasing network lifetime. The major portion of energy consumption of a sensor node is due to data transmission between nodes. Since at the present time, most wireless applications are operating in shared unlicensed spectrum bands (2.4 GHz ISM band), this band of frequency is becoming overcrowded and congested. It leads to a reduction in the quality of communications. This quality reduction makes a significant increase in packet loss and drop caused by collisions, packet retransmissions. This causes a lot of energy to be wasted. To overcome this loss of energy sharing licensed spectrum bands between unlicensed devices is an appropriate solution.

Heterogeneous sensor network consists of a group of sensor nodes with different ability, such as different computing the range of power and sensing. Compared with homogeneous WSN, deployment and topology control are more complex in heterogeneous WSN. For deployment of these sensors, a deployment and topology control method is developed. It is based on the unequal sensor model used to approximate the behaviour of sensor nodes. Besides, a cost model is planned to evaluate the deployment cost of heterogeneous WSN[5].

\section{EXISTING SYSTEM}

Conventional approaches to mobile sensor deployment are specifically designed for Homogeneous sensors and not for Heterogeneous sensors. For deployment of these heterogeneous sensors VorLag algorithm was used which was a little bit energy consuming and Sensor self-organisation was not discussed. Deploying of mobile sensors are based on centralised approaches i.e., powerful cluster head is available to collect the sensor location ${ }^{[1]}$.

It is known from existing works that WSNs were used in outdoor and indoor environments, it is difficult to deploy sensor in outdoor and easy in indoor ${ }^{[7]}$. Many works were carried out to address energy conservation issue in indoor environment. Required amount of sensors were spaced randomly for monitoring and to ensure redundancy, to design a sensor node for the above problem the sensor should switch between two modes ie., active to sleep and sleep to active mode.

\section{PROPOSED METHOD}

Sensor deployment is planned and it is possible for monitoring environments, various static deployment strategies have been introduced to enhance the surveillance coverage. In this kind of research studies, one of the most commonly considered metric is to minimize the number of sensors required to achieve some sensing coverage. Due to different sensor capabilities and manufacturing expenses, this metric is sometimes transformed into optimizing the required total device cost for those deployed sensors, making this research subject more interesting yet challenging. However, such static deployment involves manual sensor placement and is incapable of dynamically repairing sensing voids (uncovered areas) in the presence of unexpected sensor failures.

Accordingly number of efforts have been explored the movement-assisted sensor deployment techniques by utilizing mobile sensors to enhance the sensing coverage after an initial random placement of sensors. With the motion facilities equipped at the sensing devices, sensors can move around to deploy themselves. Given any number of randomly placed sensors, in the authors present a centralized force-guided algorithm motivated by the disk packing theory and virtual force field concept from robotics, to establish motion paths for 
sensors. Assuming there exists a powerful cluster head, capable of communicating with all sensors and obtaining sensor locations, the proposed algorithm evaluates all attractive and repulsive forces and obtains the resultant force applied on each sensor. The computed resultant force then directs the sensor to move to a desired position. We consider smart sensors with mobility capability to accomplish self-deployment after an initial arbitrary placement of sensors. Furthermore, since sensing devices are prone to errors due to energy depletions or unexpected failures, faulty sensors may occur over time, leaving monitoring voids (uncovered sensing holes). With the movement ability, instead of replacing faulty sensors with new ones, those smart sensors reposition themselves to restore the sensing coverage[2].

\section{A. Coverage - aware sensor automation protocol:}

Two deployments related mechanisms are combined in our CASA protocol set: EVFA-B and SSOA. Detailed processes of respective mechanism with the objective of improving the sensing coverage for a smart sensing environment. Below we summarize the environmental assumptions made in this work.

A discrete coordination system is adopted for monitoring sensing field in the network. Locations of all sensors are obtained via the pre-deployed RFID platform or some existing localization technique, and constantly updated to the cluster head[8]. Neighbouring nodes under the adopted coordination system are defined as sensors within the sensing range, which is normally much smaller than the radio communication distance. Without loss of generality, we assume that in our model. According to the derivations in the radio communication range is at least twice the sensing radius, complete coverage of a convex area implies connectivity among the working set of sensor nodes. Consequently, in this work, we only deal with the sensing coverage, and network connectivity.

\section{B. Enhanced Virtual Forces Algorithm with Boundary Forces (EVFA-B):}

The idea behind virtual forces is inspired by the combined idea of potential field and disk packing theory. Each sensor behaves as a source giving a force to others. This force can be either attractive or repulsive. If two sensors are too close, they exert repulsive forces to separate each other, otherwise they exert attractive forces to draw each other. We quantify the definition of "closeness" by using the distance threshold for any two sensors si and sj with respective sensing radius ri and rj. Given $\mathrm{k}$ sensors (denoted as $\mathrm{s} 1, \mathrm{~s} 2, \ldots$, sk with sensing radius $\mathrm{r} 1, \mathrm{r} 2, \ldots$, rk respectively) placed in the monitoring area, for any two sensors si and sj located at coordinates (xi, yi) and (xj, yj), we adopt the Euclidean distance dij to indicate how far the two sensors are spaced, where

$$
d i j=\sqrt{(x i-x j)^{2}+(y i-y j)^{2}}
$$

As a result, if $\mathrm{dij}>\mathrm{d} \mathrm{ijth}$, then attractive force is applied. On the other hand, repulsive force is generated if $\mathrm{dij}<\mathrm{d}$ ijth.

\section{Sensor Self-Organizing Algorithm:}

Wireless sensors are fundamentally unreliable. Due to sensor energy reductions or unexpected failures, the decreased sensing coverage depreciates the event detection capability of a WSN. To preserve the required sensing cover- age, one alternative is to perform EVFA-B periodically for global redeployments. However such constant global redeployment is costly in terms of communication overhead and spent moving energy, and should be kept occasional. Therefore, we propose the SSOA to firstly repair the sensing void by locally relocating sensors around the sensing hole. Two issues will be addressed to realize this local recovery: selection of repairing sensors and physical movements performed by the selected sensors. In case the local repairing is unable to recover the required detection capability, SSOA then invokes EVFA-B to globally redeploy sensors.

- Local Selection of Rescue Sensors:

The first challenge of completing partial repair is to locally select the rescue sensors around the sensing hole. Given a sensing hole caused by some broken sensor (Sdead), all active sensors nearby (not necessarily the immediate neighbours of Sdead) can be potential candidates to perform the local repair. Theoretically, every combination of rescue sensor candidates along with various moving approaches should be examined to obtain the most desirable coverage improvement. However, this approach is intractable, and not implementable. Therefore, we limit the search of rescue sensors to the neighbouring nodes of Sdead, defined as set Ndead. Our objective is to select a subset Rdead of local rescue sensors from Ndead for repairing the sensing hole.In order to evaluate the recovering capability of each sensor, we try to quantify the overlapping degree possessed by each sensor, and associate an overlapping weight wi with sensor, overlapping degree wij is defined as the overlapped area between the two sensors. Then the overlapping wi can be approximated by summing up overlapping weights contributed from all neighbours. Though, bearing in mind the existing sensing hole(s) around si, the overlapping degree should be adjusted by deducting the uncovered area(s) from wi to reflect this fact. As a result, the quantified overlapping degree wi can be either positive or negative, reflecting the recovering capability of rescue sensor.

From extensive experiments conducted, we observe that selecting adjacent sensors to move simultaneously usually leads to unnecessary overlapping and cannot successfully cover the sensing hole. On the contrary, selecting non-adjacent sensors, to cooperatively repair the sensing void generally produces effective coverage. The results recommend that the impact of locations of selected salvage sensors seems to be more pronounced than that of overlapping degrees. Consequently, in this work, we propose to select a salvage sensors set Rdead that contains non-adjacent sensors in Ndead with the highest $\mathrm{w}$ value.

\section{- Physical Movements Performed by Selected Rescue} Sensors

Once the salvage sensors set Rdead is determined, we propose to perform two-tier physical movements to gradually recover the sensing hole. After performing the first-tier movement, some neighbours of sr may become disconnected. For those affected immediate neighbours of sr, we suggest to apply the second-tier movement. Suppose sri represents some disconnected neighbour of sr. The cluster head then instructs sri to move toward sr by the amount of offset. All affected immediate neighbours of sr should perform the second-tier movements.

One may contend that more-tier physical engagements with gradually decreased movement amounts should be performed. However, this complicates the computation, and does not produce significant coverage enhancement in our experiments. As a result, we limit our local renovating within two tiers. Further evaluations of this parameter will not be included in the current work. For cases that are beyond the retrieval ability of two-tier movements due to insufficient sensors available around the sensing hole, we simply activate EVFA-B for global redeployments.

\section{- SSOA Algorithm Summary:}

Experimental understandings reveal that the local recovery mechanism exercised by SSOA provides the network an effective self-healing capability in many faulty cases, where faulty sensors are generally consistently distributed across the network. In extreme cases, where sensor faults are concentrated at certain locations, leading to a reduced sensing coverage 
below cth even after the local repairing is completed, then EVFA-B should be utilized to globally redeploy the sensors.

\section{Performance Evaluation:}

Here we validate the proposed CASA protocol by comparing the performance with other self-deployment mechanisms in terms of coverage ratio, monitoring density, network selfhealing capability, and energy consumed by sensor physical movements. For global deployments, the comparison targets include two mechanisms based on virtual forces and one based on the Voronoi diagram. We implement virtual-forces-based Zou and Zou-B with fixed weight settings. When faulty sensors occur, Zou, Zou-B, and VorLag have no local recovery technique and can only per- form global redeployments on triggered by the reduced coverage lower than cth, whereas CASA is able to quickly react to the faults by constantly applying SSOA for local repairs. For comparison purposes, we implement two other local-repairing mechanisms, namely $\mathrm{Co}-\mathrm{Fi}$ and PSO besides our pro- posed SSOA protocol, to validate the network self-healing capability in Section 6.3. When a sensor node fails, the coverage fidelity $(\mathrm{Co}-\mathrm{Fi})$ algorithm selects one single sensor (with the best/maximum utility) among neighbouring nodes around the faulty sensor to repair the sensing hole. The PSO algorithm uses a particle swarm optimization technique to rearrange neighbouring sensors involved in the fault region so that a maximum/optimized local coverage can be provided. We simulate heterogeneous sensors, having sensing radius uniformly distributed in a rectangular grid-based monitoring area[6].

\section{- Improved Surveillance Coverage:}

For smart sensing surroundings, the network typically starts with an irregular deployment of sensor nodes on the other hand, due to lack of boundary forces, Zou makes many unnecessary movements beyond the sensing field. By incorporating the boundary forces to keep sensors from drifting away, Zou-B outperforms Zou as a result of reducing unwanted coverage outside the monitoring region. On the other hand, due to improper distance threshold and weight settings, Zou-B is unable to cover the area as effectively as CASA does. We observe that the performance of VorLag greatly depends on the initial distribution of sensor nodes. VorLag is only capable of moving peripheral sensors outwards based on constructed Voronoi-Laguerre polygons for making the movement verdicts, while leaving inside sensors with little movements. In areas with a great number of sensors closely deployed, the constructed VorLag polygons become too concentrated, leading to an extreme overlapping of sensors. Overall, this results in some sparse regions and other congested areas produced by the VorLag approach, which suggests that the performance of VorLag is restricted and only effective in certain scenarios. In contrast, our CASA deployment strategy reaches the highest coverage ratio for both cases. We observe that the achieved coverage ratio of CASA increases gradually as number of sensors grows. The reason is attributed to the judicious designs of distance threshold and weight constants, making the deployment strategy adopted by CASA adaptive to environmental parameters. On the other hand, Zou and Zou-B do not have steadily increasing sensor population, due to their improper parameter designs, the two methods made incapable of utilizing the benefit brought by increased number of deployable sensors. VorLag produces low coverage ratios and is also unable to steadily increase the achieved coverage as sensor population grows. Due to the algorithmic nature of VorLag, sensors distribution causes a great number of sensors to present in the same VorLag polygon, leading to an insufficient coverage performance over the Monitoring area.
- Moderate Monitoring Density:

In addition to that we study the coverage level achieved by respective mechanism. Define the coverage density parameter $\mathrm{d}$ as the degree/level a piece of area is covered/monitored by sensors. A zone with coverage density $\mathrm{d}>1$ infers that it will not be left unmonitored once a single sensor in this area becomes un-functional. Therefore, the cover- age level in some sense indicates the fault-tolerance ability of a sensor network. However, a high coverage level (sensing redundancy) means more sensors should be deployed with high overlapping degree, which directly affects the entire sensing coverage ratio under limited number of deployable sensors. Therefore, high coverage degrees are not always beneficial. Rather, achieving desirable sensing coverage with moderate coverage level is

Coverage performance accomplished by Zou, Zou-B, VorLag, and our CASA deployment strategies under various amounts of sensor nodes in a monitored area with different initial configurations. Note that the results are taken after the first redeployment of occasional sensor faults. This further corroborates the effectiveness and robustness of our CASA operations.

\section{- Network Self-Healing Capability:}

Once the preferred sensing coverage is realized by the first redeployment, how to maintain effective surveillance coverage as faulty sensors occur over time is an important issue. We do some set of experiments to combine four global deployment strategies (Zou, Zou-B, VorLag, and EVFA-B) with three localrepairing algorithms (Co-Fi, $\mathrm{PSO}$, and $\mathrm{SSOA})$ to compare their performances. We also implement the stationary mechanism, which does not execute network healing even in the face of faulty sensors, for comparison purposes. We investigate the network self-healing issue by simulating an environment where faulty sensor occurs at every time unit. We observe the attainable coverage ratios under all combination, SSOA always sustains the longest network lifetime, exhibiting the best localrepairing capability to be combined with respective global deployment algorithm. CASA consumes less energy than three other mechanisms, Zou, Zou-B, and VorLag for the latter three mechanisms repeatedly do global redeployments, which consume significantly more energy than local-repairing schemes. During the simulation, Zou and VorLag execute global redeployments at all times since their coverage ratios never reach cth (95 percent), while Zou-B calls for global deployment at time unit of 10 (when coverage ratio goes below cth).

\section{- Energy Efficiency on Physical Movements:}

The coverage - aware energy consumption as energy spent for making one percent of coverage development. We conduct experiments to observe the energy efficiency under different sensor distributions and populations. The comparison of results of both coverage-aware energy consumption and achieved coverage ratio gives detailed output definition. The three compared mechanisms, Zou, Zou-B, and VorLag never attain coverage relations above 80 percent under all sensor inhabitants, whereas CASA is able to reach a coverage ratio of around 95 percent when $\mathrm{k}^{1 / 4} \mathrm{9}$. In this figure, VorLag put away the least energy but makes little development in the sensing coverage ratio. CASA yields the highest coverage ratio, while consuming a relatively moderate amount of energy on physical schedules, due to its ability of keeping sensors from moving far away. The results indicate that CASA is both coverage effective and energy efficient, which encourages us to implement the CASA protocol suite in a practical monitoring testbed.

\section{- Implementation of an Automated Monitoring Network:}

The simulation models do not sufficiently capture the radio and sensor irregularity in a real- world environment, a proof-ofconcept implementation is thus needed to demonstrate the feasibility of our recommended CASA protocol. Here we briefly 
discuss our proto-typing involvements on an automated MoNet enabled by CASA. Fig.4.2 illustrates the hardware architecture and communication protocols used by our MoNet. The mobile sensor is basically a moving robot carrying a single-board computer, a sensor-equipped mote, and a webcam device.

The server acts as the cluster head performing deploymentrelated computations required by CASA, while the data collector is responsible for gathering necessary data (such as sensor locations and sensing ranges) from all sensors through ZigBee and providing them to the server. In our testbed, the locality information is obtained via a pre- deployed RFID positioning system with grid granularity of $1 \mathrm{~cm}$. To demonstrate the emergency response capability of MoNet, we randomly place six mobile sensors in a $2 \mathrm{~m} \times 2 \mathrm{~m}$ area, and generate four emergency events at the four corners. We configure the sensors to regard a light event with analysis above 900 as an irregular event and report the detected event back to the server upon the detection. In addition, we simulate faulty sensors by turn-off $\mathrm{s} 1$ and $\mathrm{s} 2$ at demo time snapshots $\mathrm{t} 1$ and $\mathrm{t} 2$ respectively, leading to more detection holes as time advances, to test the network self-healing competency.

\section{RESULT AND DISCUSSION}

The coverage recovery system proposed by CASA yields the highest coverage ratio, while consuming a relatively moderate amount of energy on physical movements, due to its capability of keeping sensors from moving far away. The table 6.1 shows that the CASA protocol has advantage over other existing algorithms. The results indicate that CASA is both coverage effective and energy efficient, which encourages us to implement the CASA protocol suite in a practical monitoring testbed.

Table.6.1: Comparison table

\begin{tabular}{|c|c|c|}
\hline $\begin{array}{c}\text { Types of } \\
\text { Algorithm }\end{array}$ & $\begin{array}{c}\text { Energy } \\
\text { consumption }\end{array}$ & $\begin{array}{c}\text { Coverage } \\
\text { ratio }\end{array}$ \\
\hline CASA & 1.605 (joules) & $>=95 \%$ \\
\hline ZOU, ZOU-B & 2.005 (joules) & $<80 \%$ \\
\hline VorLag & 2.900 (joules) & $<80 \%$ \\
\hline
\end{tabular}

The fault node is identified by the lack of acknowledgment from the neighbour and these neighbour nodes are indicated as struck nodes which are highlighted in pink colour for differentiating it from the other node. The defective node is indicated using black colour. This can be noted from the Fig.6.1.

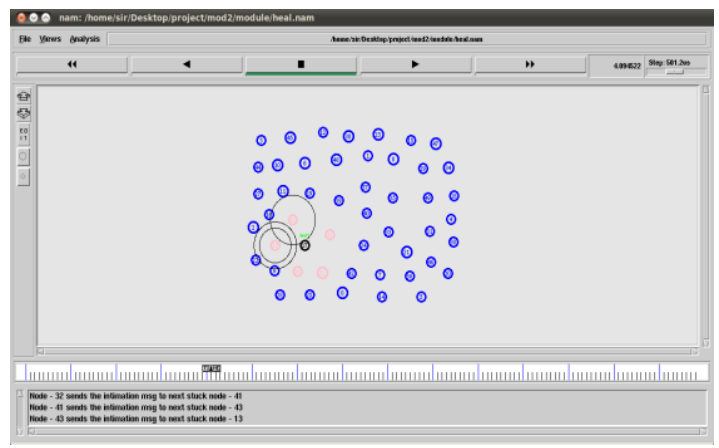

Fig. 6.1: Identification of faulty node and acknowledges the neighbour nodes.
The comparison of CASA protocol with the other traditional method in terms of coverage ratio and energy consumption is graphically represented in Fig.6.2, Fig.6.3, and Fig.6.4.

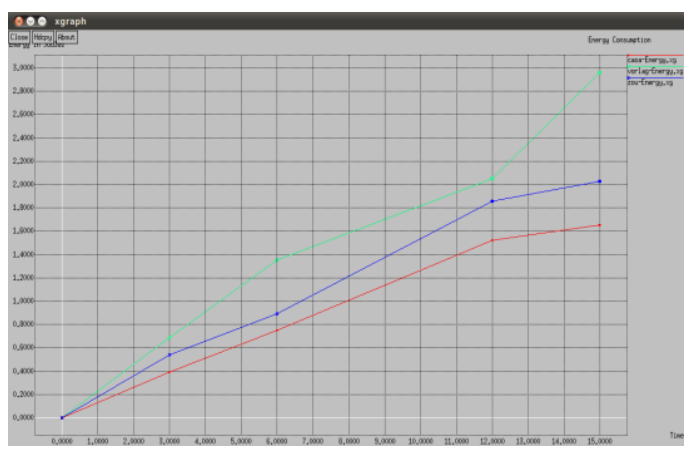

Fig. 6.2: Coverage ratio vs No. of sensors with respect to time

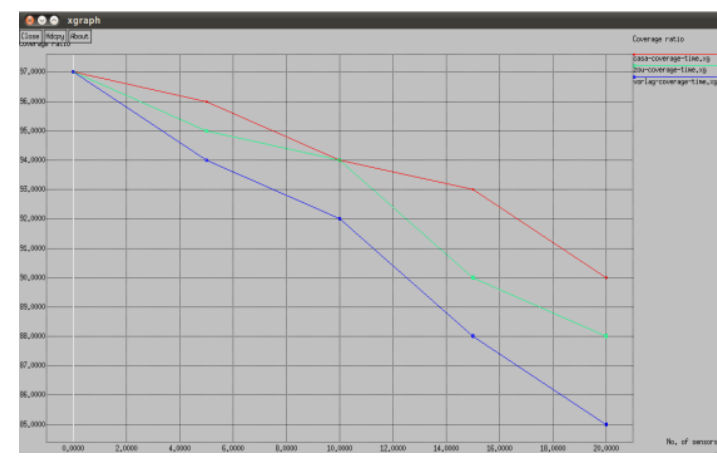

Fig. 6.3: Coverage ratio vs No. of nodes with respect to distance

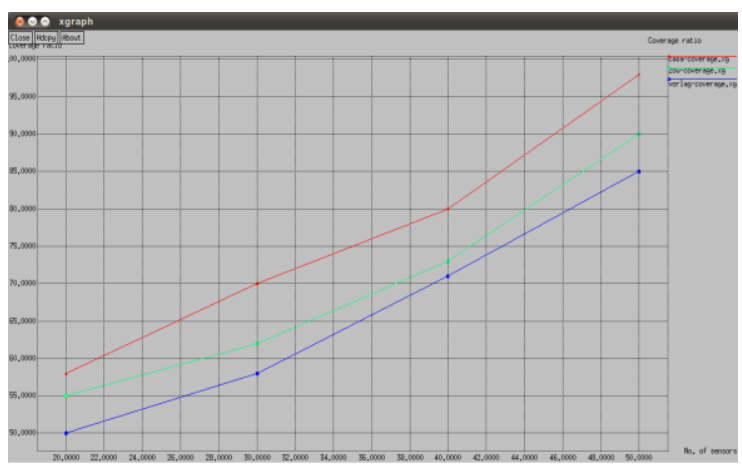

Fig. 6.4: Energy consumption vs Time period

\section{CONCLUSION}

A CASA protocol with the goal of providing effective surveillance coverage for smart sensing environments is proposed. Two centralized algorithms are included in the CASA protocol suite, viz. EVFA-B and SSOA, to independently handle the global sensor self-deployment and local sensor self-organization in the presence of node failures. The comparison table in the result shows that the CASA protocol consumes less energy and provides large coverage ratio. An automated MoNet powered by CASA protocol suite is implemented as a proof-of-concept prototype to corroborate the protocol feasibility and demonstrate the emergency detection capability of MoNet. Renewable batteries can be used to charge the wireless sensors instead of old system, can be extended to space research like weather forecasting, GPS/GPRS system. 


\section{REFERENCES}

[1] Ting - Yuin, Hendro Agus Santoso, Kun - Ru Wu, "Global Sensor Deployment and Local Coverage - Aware Recovery Schemes for Smart Environments", IEEE Transaction on Mobile Computing, vol. 14, no.7, July 2015.

[2] Xbee sensors specification [online]. Available :htttp:// digi. com/ pdf/ ds- xbeesensors.pdf.

[3] Wireless Sensor Networks An Information Processing Approach, Feng Zhao-Microsoft Corporation, Leonidas J.Guibas - Stanford University.

[4] N. Bartolini, T. Calamoneri, Thomas F. La Porta, and S. Silvestri, "Autonomous deployment of heterogeneous mobile sensors," IEEE Trans. Mobile Computing, vol. 10, no. 6, pp. 753-766, Jun. 2011.
[5] Y.-C. Wang, C.-C. Hu, and Y.-C. Tseng, "Efficient placement and dispatch of sensors in a wireless sensor network," IEEE Trans. Mobile Computing, vol. 7, no. 2, pp. 262-274, Feb. 2008

[6] Q. Wang, K. Xu, G. Takahara, and H. Hassanein, "Device placement for heterogeneous wireless sensor networks: Minimum cost with lifetime constraints," IEEE Trans. Wireless Communication, vol. 6, no. 7, pp. 2444-2453, Jul. 2007.

[7] G. Wang, G. Cao, and Thomas F. La Porta, "Movementassisted sensor deployment," IEEE Trans. Mobile Computing, vol. 5, no. 6, pp. 640-652, Jun. 2006.

[8] K. Chakrabarty, S. S. Iyengar, H. Qi, and E. Cho, "Grid coverage for surveillance and target location in distributed sensor networks," IEEE Transaction. Comput., vol. 51, no. 12, pp. 1448-1453, Dec. 2002. 